\title{
ANALYSIS AND OPTIMIZATION OF A DEMOGRAPHIC SIMULATOR FOR PARALLEL ENVIRONMENTS
}

\author{
Vanessa Büsing-Meneses \\ Cristina Montañola-Sales \\ Josep Casanovas-Garcia \\ InLab FIB \\ Universitat Politècnica de Catalunya - \\ BarcelonaTech \\ Barcelona, SPAIN
}

\author{
Alessandro Pellegrini \\ High Performance and Dependable \\ Computing Systems Group -DIAG \\ Sapienza, University of Rome \\ Rome, ITALY
}

\begin{abstract}
In the past years, the advent of multi-core machines has led to the need for adapting current simulation solutions to modern hardware architectures. In this poster, we present a solution to exploit multicore sharedmemory capacities in Yades, a parallel tool for running socio-demography dynamic simulations. We propose to abandon the single-threaded programming approach addresses in Yades by using ROOT-Sim, a library which allows to apply discrete event simulation to parallel environments profiting share-memory capabilities. As a result of this new approach, our results show the improvement in Yades' performance and scalability.
\end{abstract}

Keywords: Parallel simulation, PDES, ABM, Social Simulation, Demography

\section{INTRODUCTION}

Nowadays, multicore/multithreaded computing systems has popularized and become the standard for high-end applications. This new architecture offers new capabilities to exploit the increase of power capacity and the memory-shared schema. In the area of parallel discrete-event simulation (PDES), the simulation process is partitioned in multiple logical processes (LPs) which execute simulation events in a concurrent manner in clusters or distributed systems (Fujimoto 2000). Commonly, the simulation kernel follows a multi-process paradigm where each process hosts its local LPs and handles its scheduled events. This process is typically done in a single-threaded manner. However, abandoning this single-threaded approach in favor of a multi-threaded programming paradigm could increase the performance achieved by running parallel discrete-event simulations.

In this work, we present a solution to exploit multicore capacity to speed up simulations of large-scale agent-based demographic systems. For that, we are using Yades, a framework which helps approaching virtual experimenting techniques to modelers by simulating population dynamics of individuals and their interactions in a society (Montañola-Sales 2015). Yades is implemented using $\mu$ sik parallel simulation library which supports rollback-based optimistic synchronization (Perumalla 2005). This library adopts the process interaction world-view in which a simulation model is formed by a set of interacting (logical) processes. Logical processes (LPs) communicate through events with the standardized communications protocol Message Passing Interface (MPI). However, the multithreaded approach for resolving the inter-node communications among LPs by taking profit of the share-memory capabilities inside a multicore would improve the execution time of simulations.

After performing a MOSCA+ evaluation and software selection process (Rincón et al. 2003), we concluded that the general purpose library ROOT-Sim could be a good choice (Pellegrini 2014). The main reason is that ROOT-Sim uses a PDES paradigm with an optimistic synchronization schema and it has several functionalities that allows us to improve Yades simulator. 


\section{YADES USING ROOT-SIM}

The general purpose static library ROOT-Sim allows users to transparently parallelize a PDES simulation and solving its synchronization optimistically. It provides several interesting features such as working with the simulation state, managing its commitment, providing a set of topologies to customize communications between different LPs, and including a wide statistics module.

To test the impact of ROOT-Sim characteristics in Yades performance, we perform several experiments with a model that simulates the demographic evolution of Gambian immigration in Spain during 10 years (Montañola-Sales et al. 2014). All experiments were done in Capitano cluster at the Sapienza University of Rome. First, we analyzed the differences in sequential executions and later in parallel simulations. The possibility to run Yades in sequential is interesting for social scientists and modelers who do not often have access to clusters or High Performance Computing Systems. In Figure 1 (left) the improvement between the current and new implementation of Yades is shown with different population sizes per region/LP. For example, when simulating 40,000 families we obtain a gain of 8 times. Figure 1 (right) shows the speedup of our solution in parallel when varying the number of processors (threads) and LPs, while we increase the total population size. In this case, we set the migration probability to $10 \%$. As we can see, the scalability of the application increases as we simulate more regions.
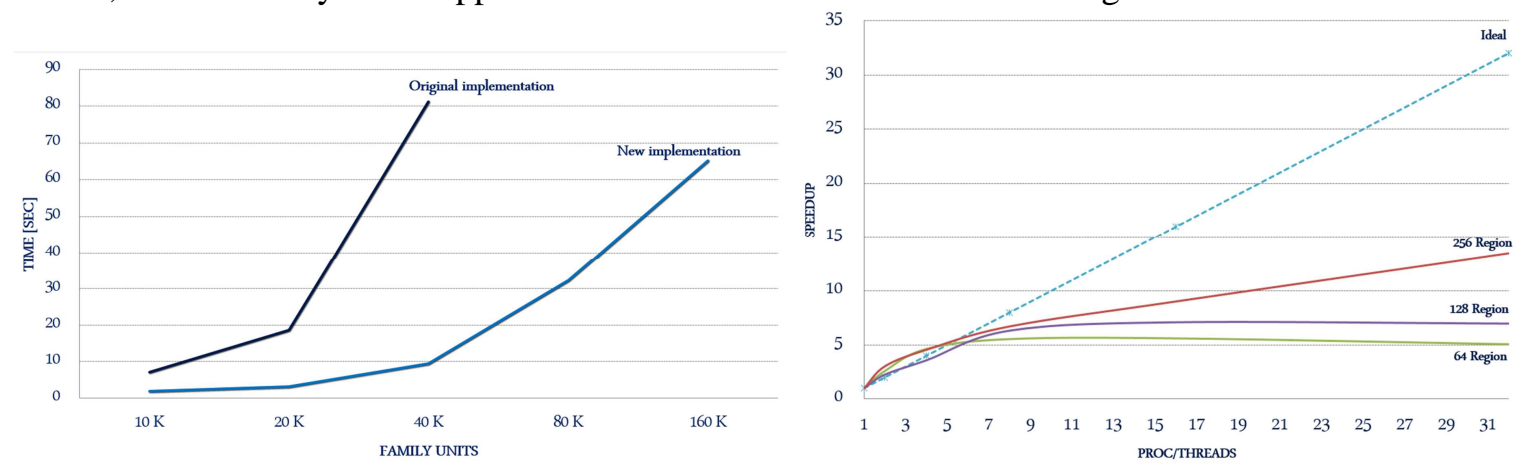

Figure 1: Comparative of Yades with previous and new implementation using ROOT-Sim in sequential (left) and parallel (right).

These results encourage us to continue to explore mechanisms to benefit from share-memory capabilities to perform large-scale demographic simulations. Further work will include to explore new schema of space distribution (based on social contacts) to measure their impact in the performance.

\section{REFERENCES}

Fujimoto, R M. 2000. Parallel and Distributed Simulation Systems. Hoboken, New Jersey: John Wiley \& Sons.

Montañola-Sales, Cristina. 2015. "Large-Scale Simulation of Population Dynamics for SocioDemographic Analysis." Universitat Politècnica de Catalunya - BarcelonaTech. http://hdl.handle.net/10803/299207 [Acessed July 27, 2015]

Montañola-Sales, Cristina, Josep Casanovas-Garcia, Adriana Kaplan-Marcusán, and Jose M Cela-Espín. 2014. "Demographic Agent-Based Simulation of Gambians Immigrants in Spain." In Advances in Computational Social Science and Social Simulation, Proceedings of the Social Simulation Conference, Barcelona, 1-5th Sept. 2014, Spain.

Pellegrini, A. 2014. "Techniques for Transparent Parallelization of Discrete Event Simulation Models." Sapienza University of Rome.

Perumalla, K S. 2005. " $\mu$ sik: A Micro-Kernel for Parallel/Distributed Simulation Systems.” In PADS'05 Proceedings of the 19th Workshop on Principles of Advanced and Distributed Simulation, 59-68.

Rincón, G, M Âlvarez, M Pérez, and S Hernández. 2003. "Modelo de Calidad (MOSCA+) Para Evaluar Software de Simulación de Eventos Discretos.” In Proceedings of the IDEAS, 167-77. 